\title{
Decay of Swirling Turbulent Flow in Rod-bundle*
}

\author{
Tsutomu IKENO** and Takeo KAJISHIMA*** \\ ** Nuclear Fuel Industries,Ltd \\ 950, Asashiro Nishi, 1-Chome, Kumatori-Cho, Sennan-Gun, Osaka, 590-0481, Japan \\ *** Department of Mechanical Engineering,Osaka University \\ Yamadaoka, Suita, Osaka, 565-0871, Japan
}

\begin{abstract}
An LES was carried out to clarify the mechanism of decaying swirl in a rod-bundle. In our LES, an immersed boundary method was used to treat this complex wall boundary in the Cartesian grid system. A consistent immersed boundary method and a oneequation dynamic SGS model were introduced for an accurate simulation of no-slip wall condition. The Reynolds number based on the bulk velocity, the rod pitch and the kinematic viscosity was approximately 4100. Our computational results were compared well with experimental and computational data obtained in other studies. The results were able to represent the effect of flow geometry: the flow around the mixingvanes caused the swirl with a large-scale fluctuation enhancing heat transfer; a vortex enhancing enthalpy mixing between channels was produced in the rod gap; this developing vortex and the decreasing wake promoted the decay of the swirl more strongly than in a pipe. The LES technique introduced in this study is useful for designing the spacer by predicting the effect of flow control.
\end{abstract}

Key words : Turbulent flow, Swirling flow, Vortex, LES, Immersed boundary method, Finite difference method

\section{Introduction}

Swirling flow in a rod-bundle is important for enhancing heat transfer in a nuclear reactor core. As shown in Fig.1, mixing-vanes attached at the spacer of the nuclear fuel rods produce the swirling flow by distorting the mainstream. The mainstream is parallel to the bundled rods. To improve the thermal hydraulic performance, optimizing the swirl is necessary. Numerical simulations are useful to predict the effect of mixing-vane configuration on the flow and heat.

Various efforts were made to investigate the swirl downstream of the spacer: the crosssectional flow pattern was successfully simulated by RANS ${ }^{(1)}$ and visualized by PIV ${ }^{(2)}$. We do not think however that RANS or experiment is enough for the complete explanation, because axial development of the swirl is much affected by turbulence stress. An LES successfully reproduced the geometrical effect on the flow in a bare rod-bundle ${ }^{(3)}$ : the turbulence energy was transformed from mainstream component to circumferential one strongly in the rod gap. Thus LES can be an effective tool for our current issue.

The geometry characteristic of the rod-bundle causes difficulty in high-quality grid generation, owing to an azimuthally varying channel-width as well as a complex spacer configuration. In our previous work ${ }^{(3)}$, an efficient numerical procedure was developed for the immersed boundary method proposed by Mohd-Yusof ${ }^{(4)}$; the procedure was successfully applied to an LES of the fully developed turbulent flow.

In this study, our LES technique in couple with the immersed boundary method is applied to the investigation of the geometrical effect focusing on the developing swirl. Namely, a consistent immersed boundary method and a one-equation dynamic SGS model are introduced to improve the accuracy of turbulence simulation in complicated wall-boundaries. The former 


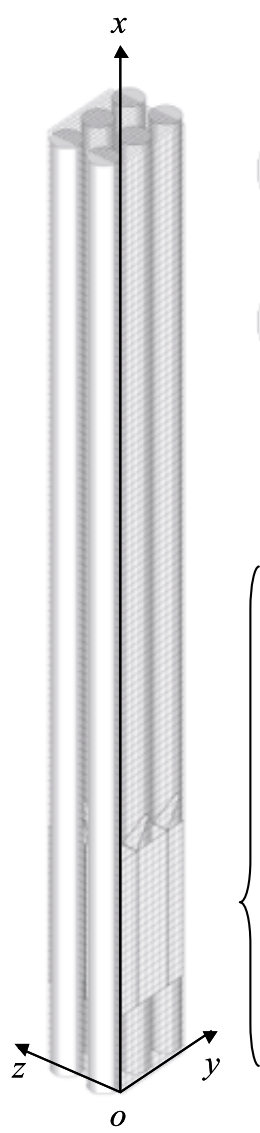

(a)

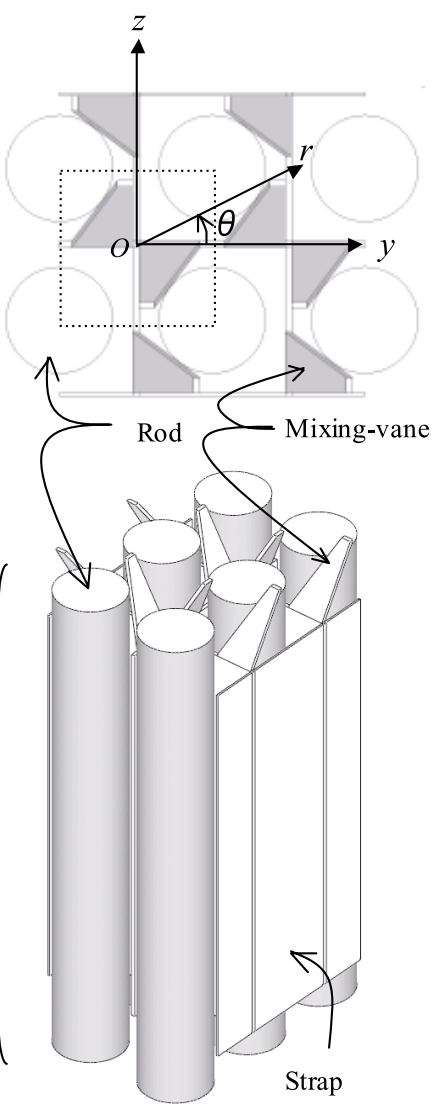

(b)

Fig. 1 The configuration of the rod bundle: (a) the computational domain and a part of grids; (b) the configuration of spacer and the unit channel (doted square) for spatial average.

maintains a consistency for the no-slip condition on the immersed wall ${ }^{(5),(6)}$. The latter is characterized by a dynamic procedure in the production term of the transport equation for SGS kinetic energy ${ }^{(7)}$.

\section{Numerical methods}

\subsection{Fundamental equations for LES}

The Navier-Stokes and continuity equations for incompressible fluid are used for the momentum and mass conservation laws. Applying the filtering operation to them results in the governing equations for the LES. By assuming an eddy viscosity model for SGS stress in the filtered momentum equation, we obtain

$$
\frac{\partial u_{i}}{\partial t}=-u_{j} \frac{\partial u_{i}}{\partial x_{j}}-\frac{\partial p}{\partial x_{i}}+\frac{\partial}{\partial x_{j}}\left[\left(v+v_{S G S}\right)\left(\frac{\partial u_{i}}{\partial x_{j}}+\frac{\partial u_{j}}{\partial x_{i}}\right)\right]+4 \delta_{i 1} u_{\tau}^{2} / D_{H},
$$

where $x_{i}$ and $u_{i}$ are respectively the coordinate distance and the filtered velocity component for the $i$-th direction in Cartesian system; $u_{\tau}$ is the averaged friction velocity; $p$ is the filtered pressure deviation from the mean value, which is divided by the fluid density; $v$ and $v_{S G S}$ are the kinematic viscosities for the molecular and SGS viscous stresses, respectively; $\delta_{i 1}$ and $D_{H}$ are Kronecker delta and the hydraulic equivalent diameter, respectively. The third term in the right-hand side represents the mean pressure gradient to drive the mainstream constantly. Equation (1) is solved in couple with the continuity equation for the filtered velocity:

$$
\frac{\partial u_{i}}{\partial x_{i}}=0
$$




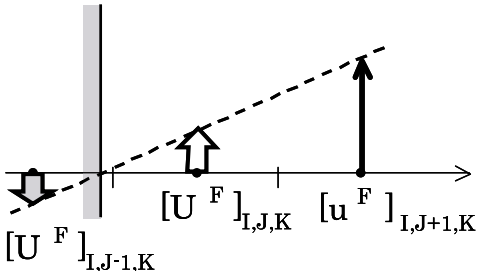

(a)

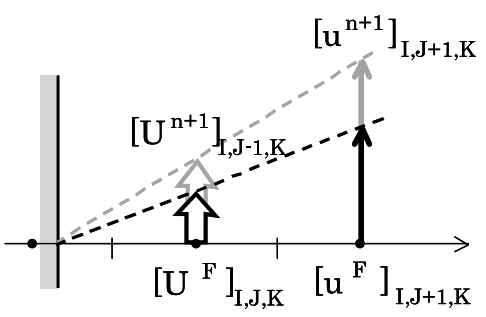

(b)

Fig. 2 The schematics of the consistent immersed boundary method: (a) the forcing method to maintain the accuracy of velocity gradient on the wall; (b) the correction method to obtain the no-slip condition at a new time step.

\subsection{Numerical scheme for the immersed boundary method}

The schematics of our immersed boundary method are shown in Fig.2. The consistency for the no-slip wall condition is maintained in the following numerical scheme.

2.2.1. The forcing method In a direct forcing method ${ }^{(4)}$, the following enforcement is applied to the right-hand side of the momentum equation:

$$
f_{i}=-R H S+\frac{\left(U_{i}^{n+1}-u_{i}^{n}\right)}{\Delta t},
$$

where RHS denotes the right-hand side of the momentum equation; $\Delta t$ is the time increment. For the no-slip condition on the wall, the desired velocity $U_{i}^{n+1}$ is given by the interpolation between zero on the wall and the value at the adjacent grid point. We write $U_{i}^{n+1}$ as follows:

$$
U_{i}^{n+1}=\varphi_{i}\left(u_{i}^{n+1}\right) \quad \text { at } \quad \mathbf{x}_{i}=\mathbf{b}_{i},
$$

where $\mathbf{x}_{i}$ and $\mathbf{b}_{i}$ indicate an arbitrary point and the forcing point, respectively; these are placed on the staggered grid point for the $i$-th flux component.

Forcing at only a grid point just outside the body ${ }^{(4)}$ decreases the accuracy of the velocity gradient on the immersed wall ${ }^{(5)}$. To avoid this, we have improved the forcing method. By applying this method of the second-order accuracy ${ }^{(5)}$ to three-dimensional geometry, the interpolation function $\varphi_{i}(\phi)$ for arbitrary flux $\phi$ is reduced as follows:

$$
\left[\varphi_{i}(\phi)\right]_{I, J, K}=\sum_{f, g, h}^{-2,2} A_{i}^{f, g, h}[\phi]_{I+f, J+g, K+h},
$$

where $(I, J, K)$ represents the grid number for $\mathbf{b}_{i}$, which is placed on the grid point nearest to the immersed wall; $A_{i}^{f, g, h}$ represents the interpolation coefficient for the $i$-th flux component. The coefficient $A_{i}^{f, g, h}$ as a function of the distance from the wall takes a value smaller than 1.0. Our method maintains the accuracy of the velocity gradient on the wall by the forcing at the grid points outside and inside the body ${ }^{(5)}$, as shown in Fig.2(a). Thus $U_{i}^{n+1}$ is interpolated by letting $f, g, h= \pm 1$ if $\mathbf{b}_{i}$ locates outside the body; it is extrapolated by letting $f, g, h= \pm 2$ if $\mathbf{b}_{i}$ locates inside the body ${ }^{(5)}$. This keeps absolute value of $A_{i}^{f, g, h}$ small enough to avoid numerical instability. The plus or minus in the double sign is chosen according to the direction from the grid point to the wall.

2.2.2. The time advancement In order to maintain the mathematical consistency between the numerical scheme and the physical no-slip condition, we have derived the following procedure for the time advancement ${ }^{(6)}$. Firstly, the fractional step $u_{i}^{F}$ is obtained by solving the following equation:

$$
\begin{aligned}
& {\left[1-\frac{\Delta t v}{2} \delta_{x_{j}} \delta_{x_{j}}-\delta\left(\mathbf{x}_{i}-\mathbf{b}_{i}\right)\left(\varphi_{i}-\frac{\Delta t v}{2} \delta_{x_{j}} \delta_{x_{j}}\right)\right] u_{i}^{F} } \\
= & {\left[1-\delta\left(\mathbf{x}_{i}-\mathbf{b}_{i}\right)\right]\left[u_{i}^{n}+\frac{\Delta t}{2}\left(3 H_{i}^{n}-H_{i}^{n-1}\right)+\frac{\Delta t v}{2} \delta_{x_{j}} \delta_{x_{j}} u_{i}^{n}+4 \Delta t \delta_{i 1} u_{\tau}^{2} / D_{H}\right], }
\end{aligned}
$$


where $\delta_{x_{i}}$ indicates the central finite difference; $H_{i}$ represents the sum of the convective term and the SGS stress term; $\delta\left(\mathbf{x}_{i}-\mathbf{b}_{i}\right)$ is a delta function. Equation(6) is based on the CrankNicolson method for the molecular viscous term and the Adams-Bashforth method for $H_{i}$. Storing the coefficient of $u_{i}^{F}$ on the computer memory reduces the computational time wasted in "IF-branching" to categorize the forcing points. This improves the efficiency of LES in extremely complex geometries.

Equation(6) represents the usual momentum equation if $\mathbf{x}_{i} \neq \mathbf{b}_{i}$. On the other hand, if $\mathbf{x}_{i}=\mathbf{b}_{i}$, Eq.(6) represents the fractional step for the desired velocity:

$$
U_{i}^{F}=\varphi_{i}\left(u_{i}^{F}\right) \quad \text { at } \quad \mathbf{x}_{i}=\mathbf{b}_{i}
$$

Then the gradient of the pressure deviation is added to advance a time step as follows:

$$
u_{i}^{n+1}=u_{i}^{F}-\Delta t\left[1-\delta\left(\mathbf{x}_{i}-\mathbf{b}_{i}\right)\right] \delta_{x_{i}} p^{n+1}-\Delta t \delta\left(\mathbf{x}_{i}-\mathbf{b}_{i}\right) \varphi_{i}\left(\delta_{x_{i}} p^{n+1}\right) .
$$

This equation maintains the no-slip condition on the wall by transforming Eq.(7) into Eq.(4). Hence, $p$ in Eq.(8) is obtained by solving the following Poisson equation:

$$
\delta_{x_{j}} \delta_{x_{j}} p^{n+1}-\delta_{x_{i}}\left[\delta\left(\mathbf{x}_{i}-\mathbf{b}_{i}\right)\left(1-\varphi_{i}\right) \delta_{x_{i}} p^{n+1}\right]=\frac{1}{\Delta t} \delta_{x_{i}} u_{i}^{F}
$$

which is derived by substituting Eq.(8) into the discrete form of Eq.(2): $\delta_{x_{i}} u_{i}^{n+1}=0$.

In Eq.(2), (6), (8) and (9), all spatial derivatives are approximated by the central difference formula of the second-order accuracy. The energy-conservation formula of the secondorder finite-difference method ${ }^{(8)}$ is particularly applied to the convective term. The time integration is implemented by the first-order fractional step method; this accuracy cannot be improved by any modification of boundary condition ${ }^{(9)}$ for fractional step $u_{i}^{F}$. Our work is not an attempt to improve such temporal accuracy but to maintain the consistency between the no-slip condition and the treatment for velocity and pressure. Figure 2(b) indicates that the consistent pressure gradient corrects the pseudo no-slip condition for the fractional step into the actual one for the new step. In our experience, a result of LES without the second term in the left-hand side of Eq.(9) indicated higher flow rate; this was caused by a flow through the immersed wall ${ }^{(6)}$.

In this study, Eq.(6) and (9) are solved by the successive over relaxation (SOR) method. This method requires many iterations until the error converges to machine-zero because the diagonal components of coefficient-matrix are modified in Eq.(9). However, we think the SOR is a good selection to minimize the computer memory requirement and to stabilize the computation.

\section{Numerical results}

In this section, the LES data set obtained for the rod-bundle geometry is verified to use it for the discussion in the next section. To simulate a whole rod bundle, the periodical boundary condition for every coordinate direction is applied to the computational domain shown in Fig.1(a). To maintain the Reynolds number around a desired value, adjusted values are given to the mean pressure gradient in Eq.(6). The following results are normalized by $P$ for length and $u_{\tau}$ for velocity.

\subsection{Drag coefficient}

In Fig.3, the drag coefficients calculated are compared with those measured. The drag coefficient for the spacer is usually based on the pressure loss ${ }^{(10)}$ calculated by subtracting friction loss in a bare rod-bundle ${ }^{(3)}$ from total loss in the rod-bundle with the spacer. We calculate the drag coefficient using the bulk velocities for this study and for the bare rod-bundle of the same computational condition. Namely, the one-equation dynamic SGS model ${ }^{(7)}$ was applied for the same mesh spacing and the mean pressure gradient. The spacer used in the experiment of Yang and Chung ${ }^{(11)}$ is a split-vane type as is simulated in our LES. Although 


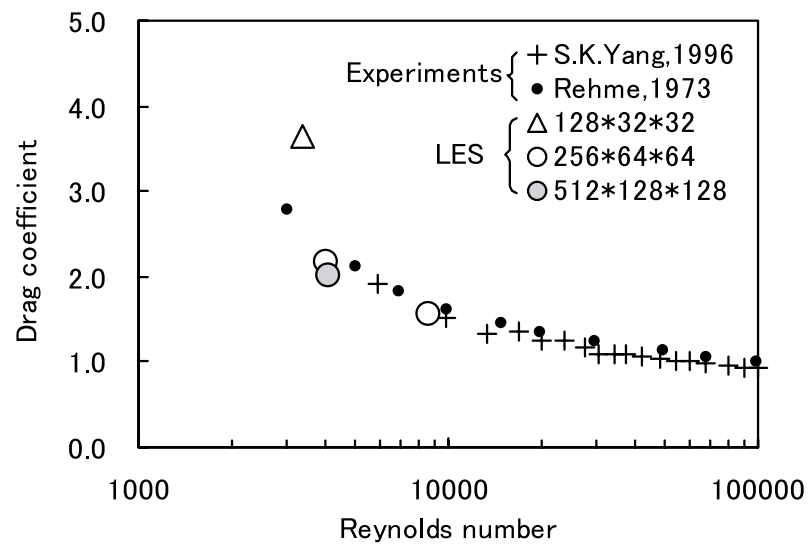

Fig. 3 Comparison of drag coefficients between LES and experiments. The grids used in LES are $128 \times 64 \times 64,256 \times 128 \times 128$ and $512 \times 256 \times 256$.

that of Rehme ${ }^{(10)}$ is a tube type, the data is useful for the comparison because it covers the low Reynolds number region.

Our numerical results converge to the experimental results as the mesh resolution becomes fine. Hereafter, we use the data for the finest mesh resolution: $512 \times 256 \times 256$ for $x, y$ and $z$ direction. The time increment is $0.0001 t^{*}$, where $t^{*}$ is the dimensionless time normalized by $P / u_{\tau}$. In that case, we confirmed the ratio between the norms of the residual and the right-hand side is approximately $10^{-5}$ in SOR method within 50 iterations. The Reynolds number based on bulk velocity $u_{m}$, rod pitch $P$ and kinematic viscosity $v$ is approximately 4100 .

\subsection{Velocity field around the spacer}

Instantaneous velocity vectors are shown in Fig.4. Figure 4(a) indicates that the swirl is caused by the flow around the mixing-vane. The swirl contains large-scale fluctuation, which affects the downstream turbulence. Structure and intensity of the downstream turbulence are important for the promotion of heat transfer on the rod surface. As shown in Fig.4(b), the velocity field around an inclined plate is captured reasonably by our immersed boundary method; any numerical disturbance is not observed even in the vicinity of the immersed boundary.

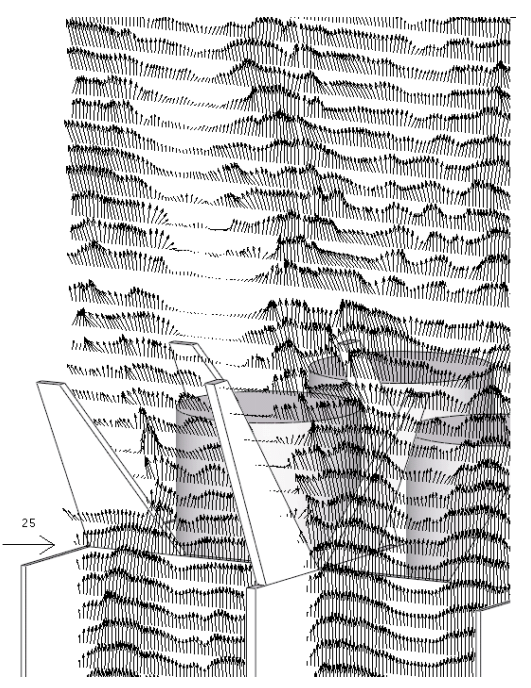

(a)

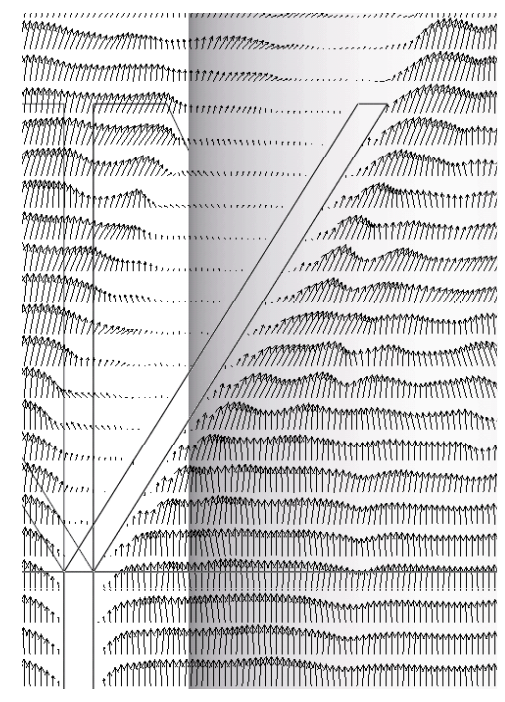

(b)

Fig. 4 Instantaneous velocities around the mixing-vane: (a) 3-dimensional vectors $(u, v, w)$ in the $x-z$ plane intersecting the mixing-vane at $y=0.18$ (plotted on every 2nd grid point); (b) 2-dimensional vectors $(u, v)$ in the $x-y$ plane intersecting the mixing-vane at $z=0.11$ (plotted on every grid point). The reference arrow indicates $25 u_{\tau}$. 


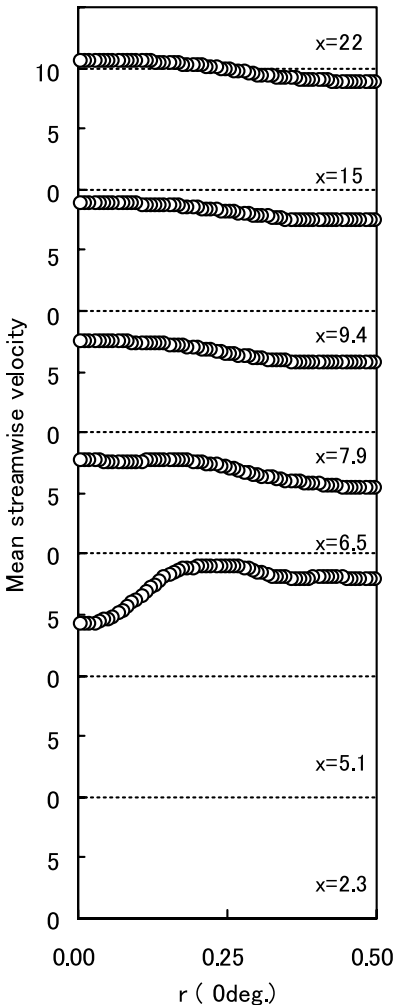

(a)

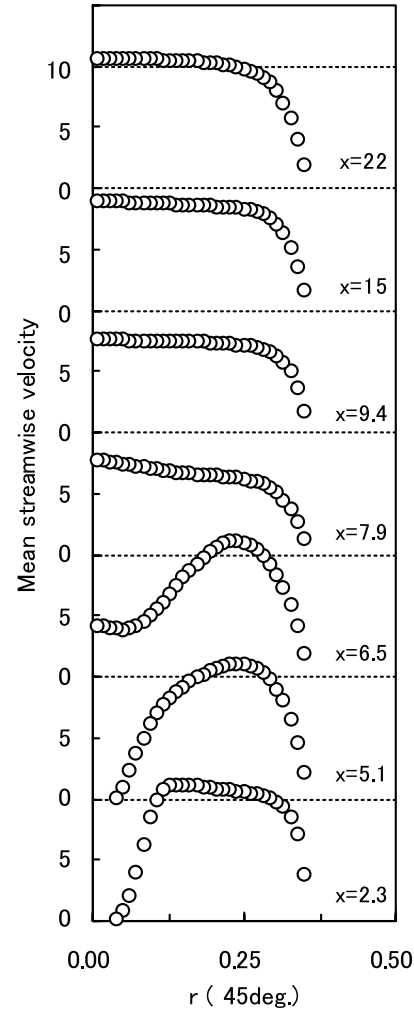

(c)

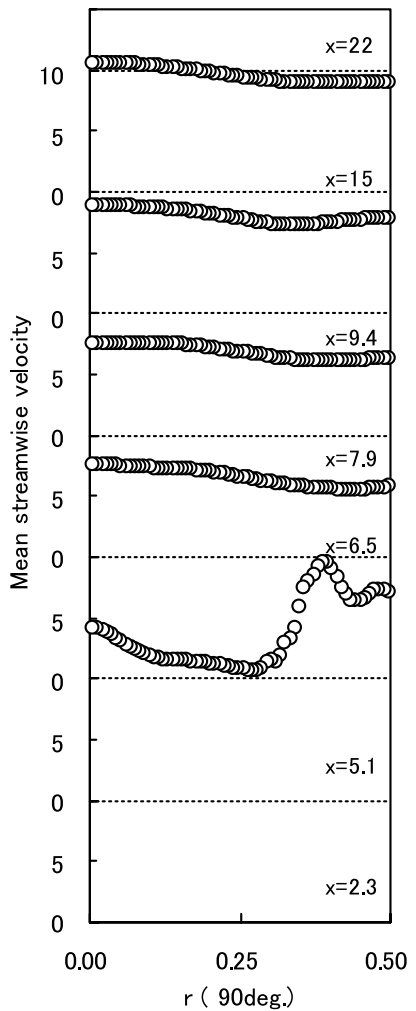

(b)



(d)

Fig. 5 Axial evolutions of the mean streamwise velocity $\overline{u_{x}}$ (normalized by $u_{\tau}$ ) in the direction of $\theta=$ (a) 0 , (b) 90 , (c) 45 and (d) -45 degrees. 


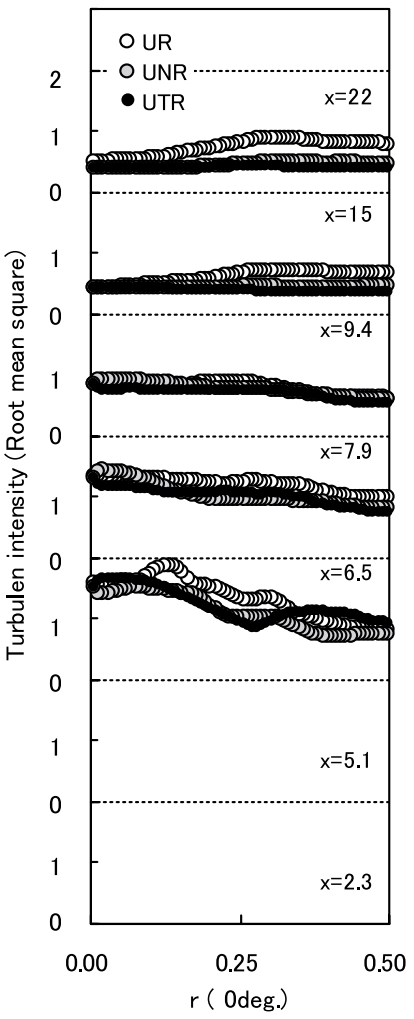

(a)

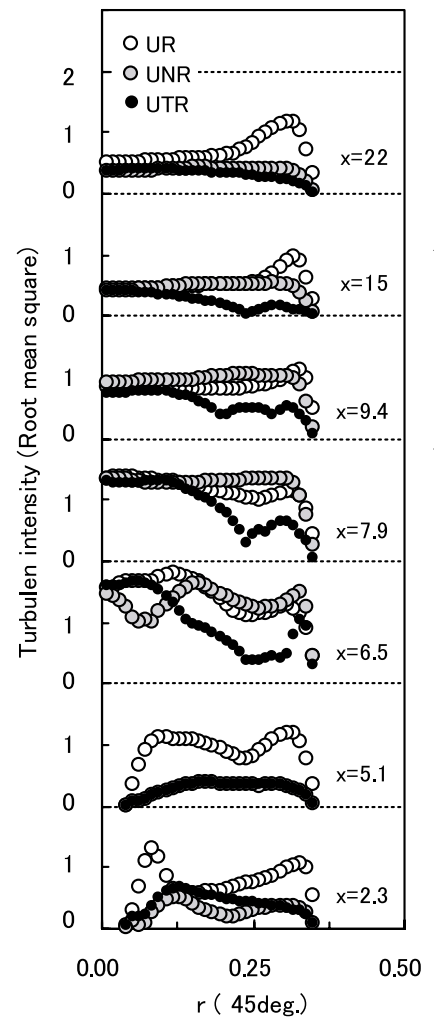

(c)



(b)

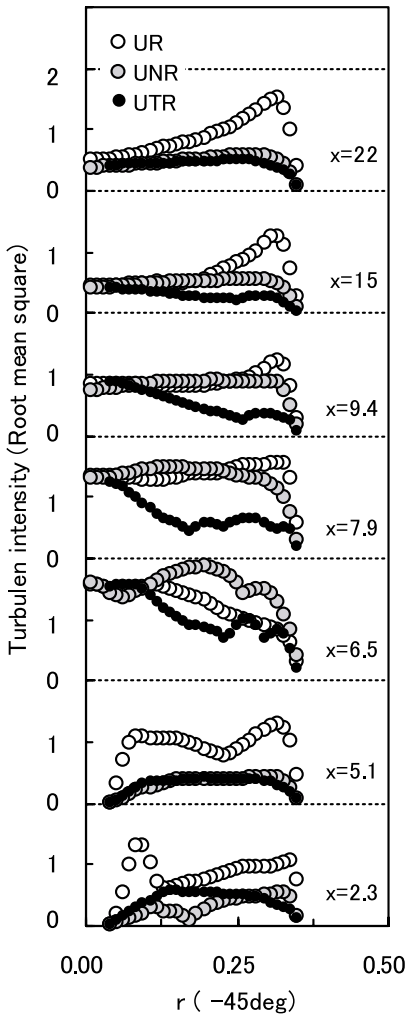

(d)

Fig. 6 Axial evolutions of the turbulence intensities $\sqrt{\overline{u_{x}^{\prime 2}}}, \sqrt{\overline{u_{r}^{\prime 2}}}$ and $\sqrt{\overline{u_{\theta}^{\prime 2}}}$ (normalized by $u_{\tau}$ ), respectively indicated by UR, UNR and UTR, in the direction of $\theta=($ a) 0, (b) 90 , (c) 45 and (d) -45 degrees. 


\subsection{Mean streamwise velocity}

Hereafter, a local coordinate system $O-x r \theta$ is applied to the unit channel shown in Fig.1(b) to discuss the mean value. To obtain the mean value, the data are averaged during $5 t^{*}$ after the flow developed, and then those for the unit channel and for the adjacent congruent channel are averaged.

Axial evolutions of the mean streamwise velocity are shown in Fig.5. In Fig.5(c) and (d), the data for $r>0.357$ are inside the rod and are not shown. The planes at $x=2.3$ and $x=5.1$ are inside the strap of the spacer. In Fig.5(a) and (b), the data inside the strap are not shown. As shown in Fig.5(c) and (d), the flow is accelerated through the suddenly narrowing channel in the spacer. The position of local maximum velocity at $x=2.3$ is close to the corner between crossing straps and that of $x=5.1$ is close to the rod surface. The plane at $x=6.5$ intersects with the mixing-vanes. In the direction of 0 and $45 \mathrm{deg}$., the velocity takes local minimum at the channel center, affected by the strap upstream. In the direction of 90 and -45 deg., the flow is the wake flow behind the mixing-vane: a local acceleration in the narrow gap between the rod and the tip of mixing-vane is simulated reasonably as shown in Fig.5(b). The planes from $x=7.9$ to $x=22$ are downstream of the spacer: in the direction of 0 and 90 deg., the profiles approach those in shear flow along the rod gap; in the direction of 45 and -45 deg., the profiles approach those in shear flow along no-slip wall.

\subsection{Turbulence intensities}

Axial evolutions of turbulence intensities are shown in Fig.6. The turbulence intensity is based on the deviation from the mean velocity. The streamwise components are dominant at $x=2.3$ and $x=5.1$. In the direction of 45 and $-45 \mathrm{deg}$, the streamwise components are remarkable in the channel center at $x=2.3$. This is caused by the imbalance between the momentum of the mainstream and the undeveloped shear turbulence stress at the beginning of no-slip wall. The imbalance becomes moderated at $x=5.1$ as the turbulence approaches a developed state. The turbulence stress is generally isotropic at $x=6.5$ : active turbulence diffusion is retrieving the velocity profile. In the direction of $90 \mathrm{deg}$., the lateral fluctuation around $r=0.3$ corresponds to the origin of the swirl near the tip of the mixing-vane. From $x=7.9$ to $x=22$, the turbulence develops with distance from the spacer.

\subsection{Decay of swirl}

Mean lateral flow pattern is shown in Fig.7, which indicates the swirls downstream of the spacer. The flow pattern is generally similar to the results of the computation ${ }^{(1)}$ and the measurement ${ }^{(2)}$. The arrangement of mixing-vanes in our LES is the same as that in Ref.(2): it isolates a swirl in the unit channel. As shown in Fig.7(a) and (b), the swirl decreases with

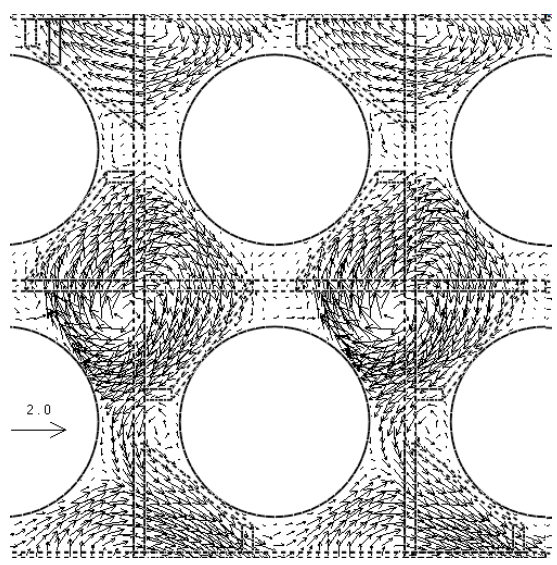

(a)

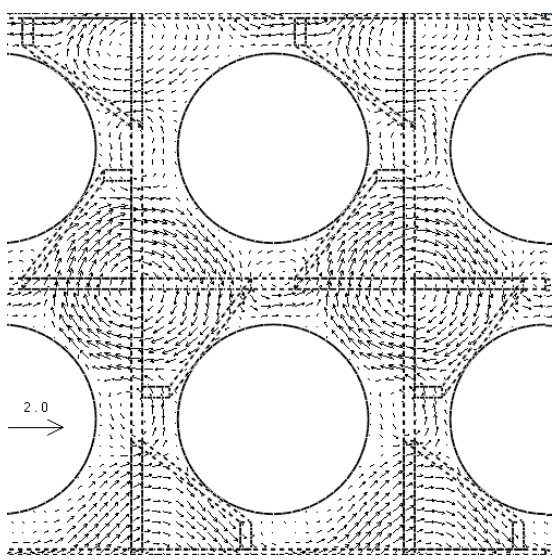

(b)

Fig. 7 The mean lateral flow pattern downstream of the spacer at (a) $x=8$ and (b) $x=16$ (plotted on every 5 th grid). The reference arrow indicates $2 u_{\tau}$. 


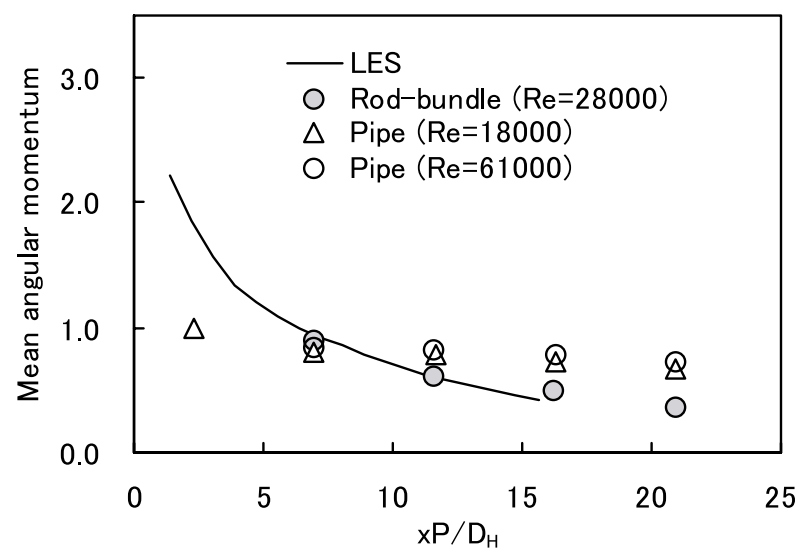

Fig. 8 Axial evolution of the mean angular momentum $\Omega$. The angular momentum is normalized by the value at $x=6.3 D_{H} / P$. The result of LES ranges from the top of mixing-vane to the bottom of strap.

Table 1 Constants for Lamb-Oseen votex (normalized by $P$ and $u_{\tau}$ )

\begin{tabular}{ccc}
\hline $\mathrm{x}$ & $\Gamma_{0}$ & $1 / 4 v t$ \\
\hline 7.9 & 2.8 & 20 \\
9.4 & 2.4 & 18 \\
15 & 1.6 & 14 \\
\hline
\end{tabular}

distance from the mixing-vanes. Predicting this decrease rate is quite important to evaluate heat transfer at the rod surface.

Axial evolution of the mean angular momentum is shown in Fig.8. In this figure, the LES result is compared with experimental results for the swirls in a rod-bundle ${ }^{(2)}$ and in a pipe ${ }^{(12)}$. The definition of the mean angular momentum is

$$
\Omega=\iint_{S} r \overline{u_{\theta}} d S / \iint_{S} d S
$$

where $S$ is the region bounded by the circle: $r \leqq 0.35$. The LES agrees well with the experiment for the rod-bundle. The decrease in the angular momentum for the rod-bundle is significant near the mixing-vanes, where comparable data is not found in the experiment. In addition, the decay of the swirl in the rod-bundle is faster than that in a pipe. We think this difference arises from the geometrical effect on the turbulence stress. McClusky, et al. ${ }^{(2)}$ investigated the lateral velocity profiles of decaying swirl in a rod-bundle by using an analogy with Lamb-Oseen vortex ${ }^{(13)}$. However, their experiment was not enough to explain such a geometrical effect. The LES can provide the filtered part of turbulence stresses. In the next section, we explain it by developing their $\operatorname{approach}^{(2)}$.

\section{Discussion}

Axial evolutions of the mean azimuthal velocity and circulation are shown in Fig.9. The data at $x=7.9,9.4$ and 15 are selected to show typical parts of developed swirl. In this figure, the results of LES are compared with those of Lamb-Oseen vortex ${ }^{(13)}$. The azimuthal velocity of Lamb-Oseen vortex is

$$
\overline{u_{\theta}}=\frac{\Gamma_{0}}{2 \pi r}\left(1-e^{-r^{2} / 4 v t}\right) .
$$

Here, $\Gamma_{0}$ and $4 v t$ represent the strength of initial source and that of viscous diffusion, respectively. Then the values of $\Gamma_{0}$ and $1 / 4 v t$ are adjusted for the curve to fit the result of LES. The adjusted values, normalized by $P$ and $u_{\tau}$, are shown in Table1. Both these values are decreasing downstream. This proposes that the geometrical effect causes the swirl to decay by decreasing its source and by increasing its diffusion. In addition, Lamb-Oseen vortex cannot 




(a)

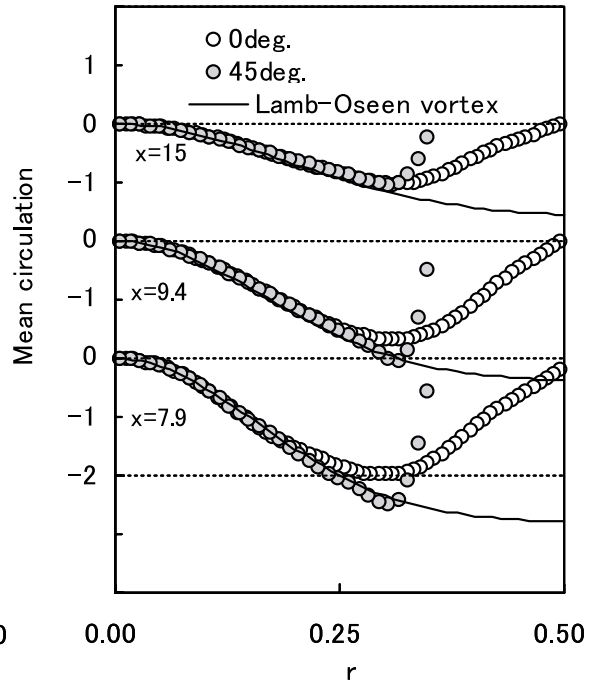

(b)

Fig. 9 Axial evolutions of the mean (a) azimuthal velocity $\overline{u_{\theta}}$ and (b) circulation $\Gamma=2 \pi r \overline{u_{\theta}}$ (normalized by $P$ and $u_{\tau}$ ).



(a)

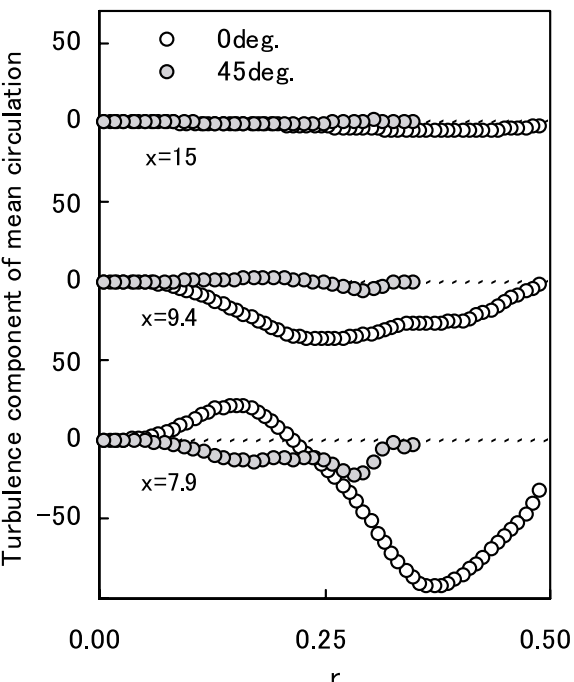

(b)

Fig. 10 Axial evolutions of the contribution of viscous and turbulence stresses to the circulation of swirl: (a) viscous stress $2 \pi r^{2} \partial \overline{u_{\theta}} / \partial r$, (b) turbulence stress $-2 \pi r^{2} \overline{u_{r}^{\prime} u_{\theta}^{\prime}} / v$ (normalized by $P$ and $u_{\tau}$ ).

represent the profiles in the outer region as shown in Fig.9. This is caused by the difference in the boundary conditions: the no-slip wall and the rod gap.

In order to consider the geometrical effect, we express the contributions of the turbulence and viscous stresses to the circulation shown in Fig.9(b). Assuming that the mean velocities are symmetric about the channel center and independent on the axial direction, the momentum equation for the mean azimuthal velocity becomes

$$
\frac{\partial \overline{u_{\theta}}}{\partial t}=v\left(\frac{\partial^{2} \overline{u_{\theta}}}{\partial r^{2}}+\frac{1}{r} \frac{\partial \overline{u_{\theta}}}{\partial r}-\frac{\overline{u_{\theta}}}{r^{2}}\right)-\frac{1}{r^{2}} \frac{\partial}{\partial r}\left(r^{2} \overline{u_{r}^{\prime} u_{\theta}^{\prime}}\right)
$$

Assuming the steady state and multiplying by $r^{2}$ derive

$$
\frac{v}{2 \pi} \frac{\partial \Gamma_{r}}{\partial r}=\frac{\partial}{\partial r}\left(r^{2} v \frac{\partial \overline{u_{\theta}}}{\partial r}\right)-\frac{\partial}{\partial r}\left(r^{2} \overline{u_{r}^{\prime} u_{\theta}^{\prime}}\right)
$$

where $\Gamma_{r}=2 \pi r \overline{u_{\theta}}$. By this definition, the circulation of the swirl shown in Fig.9(b) is represented by a negative value of $\Gamma_{r}$. Equation (13) indicates that the viscous and turbulence 
stresses cause the diffusion of the angular momentum. Integrating Eq.(13) and letting $\Gamma_{r}$, $\partial \overline{u_{\theta}} / \partial r$ and $\overline{u_{r}^{\prime} u_{\theta}^{\prime}} \rightarrow 0$ as $r \rightarrow 0$ based on the radial symmetry derive

$$
\Gamma_{r}=2 \pi r^{2}\left(\frac{\partial \overline{u_{\theta}}}{\partial r}-\frac{1}{v} \overline{u_{r}^{\prime} u_{\theta}^{\prime}}\right) .
$$

The contribution of each term in the right-hand side of Eq.(14) is shown in Fig.10. The diffusion by the viscous stress becomes evident near the rod wall in the direction of $45 \mathrm{deg}$. and in the rod gap in the direction of $0 \mathrm{deg}$.. On the other hand, the contribution of the turbulence stress indicates a significant difference in the profile between these directions.

In the direction of 0 deg., the swirl is decelerated at $x=7.9$ and accelerated at $x=9.4$, respectively owing to the negative and positive correlations $\left(u_{r}^{\prime} u_{\theta}^{\prime}<0\right.$ and $\left.u_{r}^{\prime} u_{\theta}^{\prime}>0\right)$. Since the deceleration and acceleration correspond respectively to the positive and negative fluctuations of azimuthal velocity $\left(u_{\theta}^{\prime}>0\right.$ and $\left.u_{\theta}^{\prime}<0\right)$, both the deceleration at $x=7.9$ and the acceleration at $x=9.4$ arise from the negative fluctuation of radial velocity $\left(u_{r}^{\prime}<0\right)$. This leads to the acceleration of the vortex $\left(u_{\theta}^{\prime}<0\right)$ in the rod gap (see Fig.7), where the correlation takes positive value $\left(u_{r}^{\prime} u_{\theta}^{\prime}>0\right)$. This indicates that the motion of the vortex in the rod gap is important for modeling the decaying swirl.

In the direction of $45 \mathrm{deg}$., the swirl is accelerated by the positive correlation $\left(u_{r}^{\prime} u_{\theta}^{\prime}>0\right)$. Then the acceleration $\left(u_{\theta}^{\prime}<0\right)$ arises from the negative fluctuation of radial velocity $\left(u_{r}^{\prime}<0\right)$. This corresponds to the centripetal action for the swirl and to the suction into the wake region behind the mixing-vanes (see Fig.4). Therefore, the acceleration decays immediately as the wake diffuses to disappear.

Thus, the swirl in the rod-bundle is decelerated by two mechanisms as shown in Fig.11: the acceleration of the vortex developing in the rod gap and the fast decrease of the acceleration source in the wake region. They are the main reason for the swirl to decay faster in the rodbundle than in a pipe.

\section{Conclusion}

We carried out an LES of swirling turbulent flow in a rod-bundle. In order to treat this complex geometry, we used a consistent immersed boundary method and a one-equation dynamic SGS model. The geometrical effect on the velocity field was accurately simulated by



Fig. 11 The structure of swirling flow in rod bundle. Swirl is represented by mean 2-dimensional vectors in cross-sections and low speed region by equi-surfaces of streamwise velocity $\left(u^{\prime}<3 u_{\tau}\right)$. 
means of an immersed boundary method with a consistent scheme for the no-slip wall condition. The one-equation dynamic SGS model was useful for this complex geometry because its dynamic procedure required neither a near-wall correction for SGS viscosity nor an averaging operation of dynamic parameter.

The LES results were able to represent the effect of flow geometry: the flow around mixing-vanes caused the swirl with a large-scale fluctuation enhancing heat transfer and a vortex enhancing enthalpy mixing between channels was produced in the rod gap. Here, we proposed an analysis model to express the contribution of viscous and turbulence stresses. This model was successfully used to explain the mechanism of decaying swirl in the rodbundle: a developing vortex in the rod gap and the decreasing wake behind the mixing-vanes promoted the decay of the swirl more strongly than in a pipe.

This study suggests that the LES technique becomes a useful tool for designing the spacer by predicting the effect of flow control.

\section{References}

( 1 ) In,W.K. et al, Simulation of turbulent flow in rod bundles using eddy viscosity models and the Reynolds stress model, Proceedings of the 10th international topical meeting on Nuclear Reactor Thermal Hydraulics, CD-ROM E00213 (2003-10).

( 2 ) McClusky,H.L. et al, Developement of swirling flow in a rod bundle subchannel, Transaction of the ASME, Journal of Fluids Engineering, Vol.124 (2002), pp.747-755.

( 3 ) Ikeno,T. and Kajishima,T., Large eddy simulation of fully developed sub-channel turbulence, Proceedings of the 10th international topical meeting on Nuclear Reactor Thermal Hydraulics, CD-ROM A00601 (2003-10).

( 4 ) Mohd-Yusof,J., Combined immersed boundary / B-spline methods for simulations of flows in complex geometries, Center for Turbulence Research Annual Research Briefs (1997), pp.317-327.

( 5 ) Ikeno,T. and Kajishima,T., Higher-order immersed boundary method for LES with complicated boundaries, Transactions of the Japan Society of Mechanical Engineers, Series $B$, Vol.69, No.686 (2003), pp.2177-2183 (in Japanese).

( 6 ) Ikeno,T. and Kajishima,T., Difference formula of Poisson equation consistent with an immersed boundary method, Transactions of the Japan Society of Mechanical Engineers, Series B, Vol.70, No.697 (2004), pp.2239-2245 (in Japanese).

( 7 ) Kajishima,T. and Nomachi,T., One-equation subgrid scale model using dynamic procedure for the energy production, Proceedings of the 4th ASME/JSME Joint Fluids Engineering Conference, CD-ROM 45348 (2003-7), also in Transaction of the ASME, Journal of Applied Mechanics, 73 (in press).

( 8 ) Kajishima,T., Conservation properties of finite difference method for convection, Transactions of the Japan Society of Mechanical Engineers, Series B, Vol.60, No.574 (1994), pp.2058-2063 (in Japanese).

( 9 ) Perot,J.B., An analysis of the fractional step method, Journal of Computational Physics, Vol.108 (1993), pp.51-58

(10) Rehme,K., Pressure drop correlations for fuel element spacers, Nuclear Technology, Vol.17 (1973), pp.15-23

(11) Yang,S.Y. and Chung,M.K., Spacer grid effects on turbulent flow in rod bundles, Journal of Korean Nuclear Society, Vol.28, No.1 (1996), pp.56-71

(12) Kreith,F. and Sonju,O.K., The decay of a turbulent swirl in a pipe, Journal of Fluid Mechanics, Vol.22 (1965), pp.257-271.

(13) Saffman,P.G, Vortex Dynamics, (1992), pp.253-257, Cambridge University Press. 